\title{
Numerical Studies of Divided Aperture Techniques in Confocal System
}

\author{
Haonan Lai \\ Guangzhou, China \\ Email: haolai1909@ulinkcollege.com
}

How to cite this paper: Lai, H.N. (2019) Numerical Studies of Divided Aperture Techniques in Confocal System. Journal of Power and Energy Engineering, 7, 42-46. https://doi.org/10.4236/jpee.2019.711005

Received: September 21, 2019 Accepted: November 24, 2019

Published: November 27, 2019

\begin{abstract}
This paper performs a numerical study of the imaging process in confocal microscopy. We simulate the imaging result using Fresnel diffraction formula. We also consider the divided aperture technique in confocal system. Different aperture shapes were varied to achieve best performance in terms of resolution and integrated intensity. Here, the D-shaped achieves the best performance in $\mathrm{x}$-axis resolution, while the annular aperture shape is the worst for integrated intensity. We propose using different aperture shapes for various biomedical applications.
\end{abstract}

\section{Keywords}

Confocal Microscopy, Aperture, Resolution, Integrated Intensity

\section{Introduction}

Confocal microscopy, most frequently named as confocal laser scanning microscopy (CLSM) or laser confocal scanning microscopy (LCSM), is an optical imaging technique for increasing optical resolution and contrast of a micrograph by means of using a spatial pinhole to block out-of-focus light in image formation. Compared with traditional microscopes, confocal microscopes have the following advantages. 1) Suppress the blurring of the image and obtain a clear image; 2) Because of the point-to-point scanning, the effect of stray light is removed; 3) It has higher axial resolution and can obtain continuous optical slices, that is to say, to get the image in focus at a specific depth. The image is acquired point by point and then reconstructed by computer, so it can reconstruct objects with complex topology. For opaque samples, we can use surface mapping, while for transparent samples, internal structure imaging is favored. For internal structure imaging, the image quality can be greatly improved, because the information from different depths of the sample is not overlapped. Traditional 
microscopes can "see" all the places where light is thrown. For confocal microscopes, only the information at the focus is collected. In fact, confocal laser scanning microscopy is achieved by controlling the depth and height of focus. Background signal refers to a small spurious signal in the system itself, because of the internal relationship of the system. If the sensor is not selective, it will record the signal together with the useful signal. For example, when recording, the current sound of the system is included in the audio signal, so even if there is no sound signal, the weak rustle can still be heard. This is the system's background noise. Divided aperture technique (DAT) refers to designing the shape of aperture based on the principle of angular gating. Previous studies have shown that using appropriate shape of apertures can help eliminate scattered light [1] [2]. People can control the degree of filtration by adjusting the size of the aperture. Previous studies also presented using DAT combined with modulation techniques can result in improved imaging resolution. For example, using a D-shaped aperture together with focal modulation can enhance resolution in $\mathrm{x}$ axis [3] and using annular-shaped aperture can improve y axis resolution [4]. Different resolutions bring different image quality. People can choose the right resolution for experiments with different requirements. Inspired by this, we studied different aperture shapes in this paper and compare them in terms of three-dimensional resolutions.

This paper is organized as follows. First, we build the model of confocal imaging systems via diffraction analysis. Second, we designed different aperture shapes and did numerical analysis of their impacts on imaging. Third, different apertures were compared to achieve better resolution performance in the desired direction. Last, we also optimized the aperture shapes to minimized background rejection.

\section{Theory}

Figure 1 illustrates the geometry of confocal system, which takes parallel incident

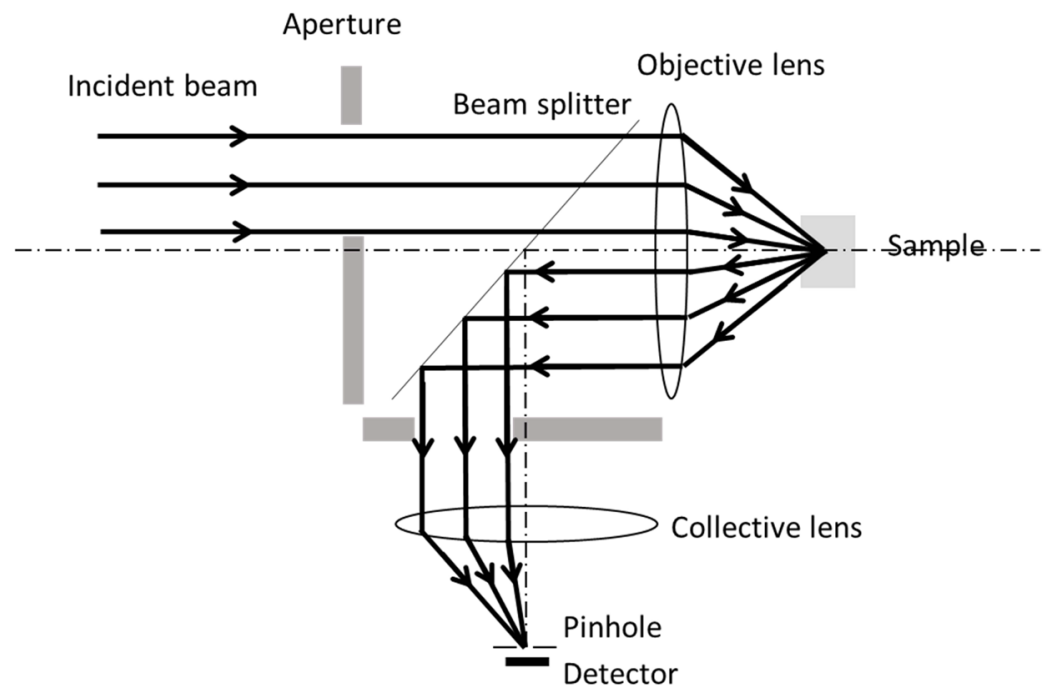

Figure 1. Geometry of confocal microscopy. Parallel incident beam is shaped by the aperture and focused to the sample via an objective lens. 
light and focus on the sample through an objective lens. The emission light is reflected by a beam splitter and focused on the detector through a collective lens and pinhole. The electric field near the focal spot can be derived as the following equation.

$$
U\left(v_{x}, v_{y}, u\right)=\frac{1}{\pi} \iint P\left(\rho_{x}, \rho_{y}\right) \exp \left\{i\left[v_{x} \rho_{x}+v_{y} \rho_{y}-\frac{1}{2} u\left(\rho_{x}^{2}+\rho_{y}^{2}\right)\right]\right\} d \rho_{x} d \rho_{y}
$$

The focal length of lens is calculated by Fresnel diffraction formula. Here the optical coordinates are related to the true distances from the focal point $\mathrm{x}, \mathrm{y}, \mathrm{z}$ by $v_{x}=2 \pi x n \sin \alpha / \lambda, \quad v_{y}=2 \pi y n \sin \alpha / \lambda, u=8 \pi z n \sin ^{2}\left(\frac{\alpha}{2}\right) / \lambda$, where $\lambda$ indicates the wavelength, $\alpha$ is the semi-angular aperture of the lens, and $n$ is the refractive index of the immersion medium. The coordinates $\rho_{x}, \rho_{y}$ are distances in the pupil plane, normalized by the pupil radius a. We expect the scalar paraxial theory to be valid for values of $\alpha<30^{\circ}$ and to give qualitatively correct behavior for larger values of $\alpha$ [1].

\section{Resolution}

In this paper, we studied different types of apertures and compare them in terms of the resolution and integrated intensity. Table 1 shows four aperture shapes: circular, D-shape, Annular and Crossing. Circular shape denotes the traditional aperture with the maximum pupil size. D-shaped aperture has a bar of width 0.2 in the middle. Light can only travel through this aperture in the upper semicircle

Table 1. Different aperture shapes in this study.

Aperture shape
Circular
D-shaped
Annular


or the lower semicircle. Annular aperture has a circular block in the middle, with radius 0.2 . Crossing has bars blocking light in both $\mathrm{x}$ and $\mathrm{y}$ axis. We think these four aperture shapes will have some influence on the imaging results.

Figure 2 shows the resolution in the $\mathrm{x}$-direction of these four different apertures. D-shape and Annular's aperture drop to the bottom at $\mathrm{x}=3.1$. The aperture of Crossing and Circular drops to the bottom when $x$ equals 3.7. The aperture of D-shape decreases fastest. Thus, in the $\mathrm{x}$ direction, $\mathrm{D}$-shape has the highest resolution. However, on the downside, D-shape aperture results in the largest side lobe.

\section{Integrated Intensity}

In confocal microscopy, the concept of the integrated intensity was introduced to quantify the background produced by a scattering medium [5] [6]. We calculate the integrated intensity as follows:

$$
I_{\text {int }}(u)=\int I\left(v_{x}, v_{y}, u\right) d v_{x} d v_{y}
$$

Here, $I\left(v_{x}, v_{y}, u\right)$ stands for the image of a point object by the microscopy. The integrated intensity is calculated by integrating $I\left(v_{x}, v_{y}, u\right)$ over the $\mathrm{x}-\mathrm{y}$ space.

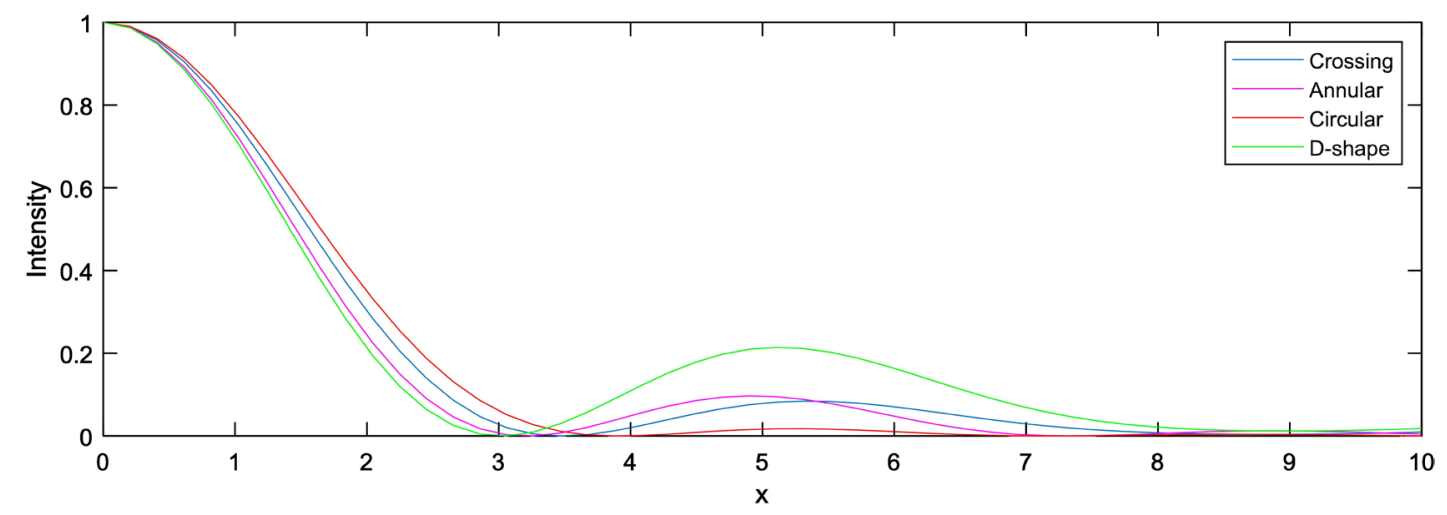

Figure 2. Resolutions in $\mathrm{x}$-axis of confocal microscopy with different aperture shapes. The D-shape aperture achieves highest resolution in this plot.

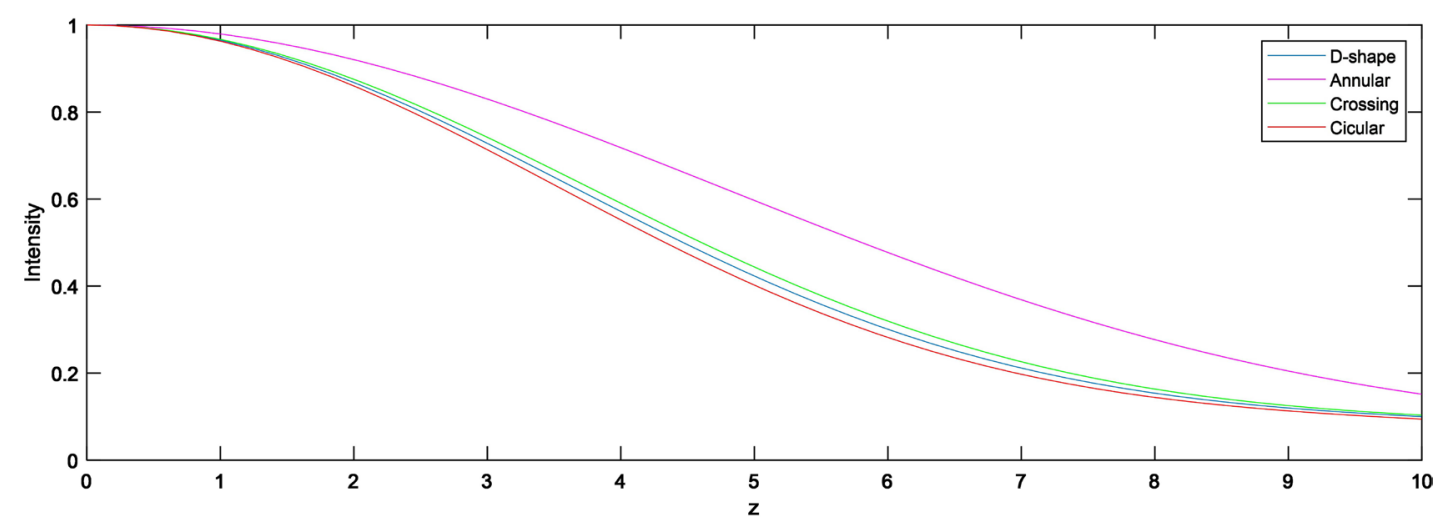

Figure 3. Integrated intensity of confocal microscopy with different aperture shapes. The annular aperture shape is the worst for this evaluation metrics. 
The $\mathrm{y}$ axis of this graph represents intensity, and the $\mathrm{x}$ axis represents the image in the $\mathrm{z}$ direction (Figure 3). Similarly, if the lines decrease faster, the microscopy has a stronger background rejection ability. In this picture, D-shape, Crossing and Circular are basically at the same level, with almost the same rate of decline. Annular shape, on the other hand, has a distinct difference. The rate of decline is a little smaller than the other three apertures.

\section{Conclusion}

In this paper, we studied the imaging of confocal microscopy in different aperture shapes. In the $\mathrm{x}$-direction, D-shape has a higher resolution. In terms of the integrated intensity, the annular aperture shape is weaker than the other three apertures. We can use these characteristics to use these apertures in different places.

\section{Conflicts of Interest}

The author declares no conflicts of interest regarding the publication of this paper.

\section{References}

[1] Sheppard, C.J., Gong, W. and Si, K. (2008) The Divided Aperture Technique for Microscopy through Scattering Media. Optics Express, 16, 17031-17038. https://doi.org/10.1364/OE.16.017031

[2] Shen, S., Zhu, B., Zheng, Y., Gong, W. and Si, K. (2016) Stripe-Shaped Apertures in Confocal Microscopy. Applied Optics, 55, 7613-7618. https://doi.org/10.1364/AO.55.007613

[3] Gong, W., Si, K., Chen, N. and Sheppard, C.J. (2010) Focal Modulation Microscopy with Annular Apertures: A Numerical Study. Journal of Biophotonics, 3, 476-484. https://doi.org/10.1002/jbio.200900110

[4] Zhu, B., Shen, S., Zheng, Y., Gong, W. and Si, K. (2016) Numerical Studies of Focal Modulation Microscopy in High-NA System. Optics Express, 24, 19138-19147. https://doi.org/10.1364/OE.24.019138

[5] Sheppard, C. and Wilson, T. (1978) Depth of Field in the Scanning Microscope. Optics Letters, 3, 115-117. https://doi.org/10.1364/OL.3.000115

[6] Sheppard, C. and Sharma, M. (2001) Integrated Intensity and Confocal Imaging through Scattering Media. Journal of Modern Optics, 48, 1517-1525. https://doi.org/10.1080/09500340108231780 